\title{
Android Based LIGHTNET for Brightness and Color Temperature Control
}

\author{
Akshada A. Hanchate ${ }^{1}$, Dr. S.B. Dhonde ${ }^{2}$ \\ Department of Electronics, Savitribai Phule Pune University, All India Shri Shivaji Memorial Society’s Institute of \\ Information Technology, Pune, India ${ }^{1,2}$
}

\begin{abstract}
These days, the LEDs used for residential, commercial and industrial sectors puposes could be configured for lively with specific lighting, which provides more efficient outcome. The basic idea is to drive the bi-color stripe (warm white stripe with cool white stripe of LEDs) for precise PWM dimming in the range of 1-10V. The dimming of LED strings is controlled by using the android phone. The most important thing that needs to be taken in consideration for controlling the light level in an energetic way, preserving the desired light level where needed while controlling light to a minimum value. However, the regulation of light intensity (brightness) and colour correlated temperature is obtained by adjusting the PWM dimming for LEDs. PWM signal enables us to attain minimum intensity levels and more linear control of light intensity compared to continuous current dimming methods. Though, accurate LEDs controlling of colors which possess color point maintenance, light intensity control along with color mixing in due with LED device lifespan and changes in temperature at a junction. Smart with efficient lighting evolves from the traditional lighting control system with feedback from integrated sensors. In order to achieve the goal of accurate color mixing of two LED strings (warm and cool white LEDs), the closed loop non-linear feedback control method is adopted instead of a linear control method.
\end{abstract}

Keywords: Bi-color LED stripe; PWM dimming; Color correlated temperature (CCT); luminance; color mixing; 0-10v range; non linear feedback control; lighting levels.

\section{INTRODUCTION}

Light emitting diode with high power possess a manifest in benefits of greater luminous efficiency, reducted energy use, [2] environment friendly and having capability of more operating lifespan over incandescent along with traditional fluorescent lamps used in numerous lighting applications. Various factors like change in component operating conditions and aging, the LEDs from the same case endowed by a manufacturer could result in inconsistent color temperature [7]. Most prominently, continuous changes in temperature during operable conditions may leads for notable differences in color appearance[8].

Now a days, different LEDs comes with various advanced techniques like large CCT (Color Correlated Temperature) adjustments and deep dimming schemes for their emitted light mixture, many research work on lighting and market sellers are blending for bi-color stripes contains warm and cool white LEDs stripes in order to get a perfect dimming and higher CCT [9] to [12].

Bi-color LEDs with dimming characteristic for adjusting CCT are evolving tremendously resulting in high-end lighting applications and various monetary worth products in the museums' ceiling lightings, lighting the market, studio lightings, photography and hotel applications, etc. For linear control systems the CCT and dimming operation ishighly inaccurate and non-independent.

The proposed system for LED control can be achieved by implementing feedback control using non-linear scheme instead of linear approach for achieving high accuracy in terms of microcontroller, color sensor, CCT and luminous values.Here, also android phone for displaying the range, Wi-Fi module for data transmission to provide the communication between microcontroller and android phone, and a bi-color LED strip. Generatinguniform and precise light outcomes by the bi-color LED course may give rise to new opportunities resulting in acquiring the high-end colormixing, enhancing the quality of dimming, and general lighting applications.

\section{LITERATURE SURVEY}

Albert T. L. Lee proposes a non linear system for with closed loop feedback method, [1] objective is to provide accurate and precise dimming for two LEDs strings comprising warm and cool white LEDs. Huan-Ting Chen defines 
color-mixing technique, possessing different LED devices of distinct color temperatures, which leads to new modelling techniques for non-similar devices. F. C. Wangpresents proposes new control theory for RGB system which is used to operate the LED color along with luminous intensity outcome. For this operation,[4] applies multivariable control techniques.Xiaohui Qu uses the light sensors with feedback techniquesin order to get the better light intensity, color(light) point, color mixing and temperature at junction in [5]. Marc Dyble intented the PWM scheme for mixed color along with phospher transformed into pure white LED outcome. [7] deals with the method used for dimming color control, current control methods for various dimming intensity of LED.M. M. Sisto overcomes the challenges faced in [7] along with it, maximation in the Color Rendering Index (CRI) which provides a accurate colors over the surface lighted by LED in [9].K. H. Loo proposes bi-level controlling application for the 2D driving of luminosity and pure white color in addition to duty cycle and current variation. In [8] the technique deals with complexity fcaing during various light interation for color shifting systems of LEDs.the theoretical aspects of these complex interactions and theimpact of bilevel drive on the color-shift properties of white LEDs.

\section{METHODOLOGY}

\section{A. Basic light theory}

Light is nothing but the electromagnetic radiation having specific wavelength. Due to the wavelength visibilityof light depends. The three primary properties of light are as follows;

i. Frequency / wavelength,

ii. Intensity (brightness), and

iii. Polarization.

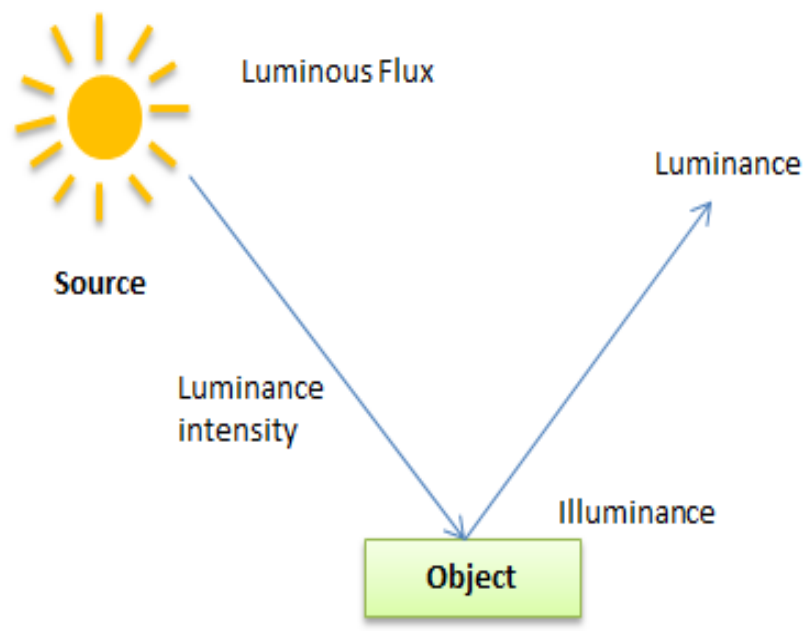

Fig. 1 Basic light terms for incident and reflected rays

Light source incident and reflected on the object provides the various parameter. Out of which the proposed system deals with 3 main concepts.

i. Illuminance: It is the overall luminous flux incidenting on an object. It is measured in $\left(1 \mathrm{~m} / \mathrm{m}^{2}\right)$, where $1 \mathrm{~m}$ stands for lumens.Illuminance represents in terms of light in a particular point of time, also expressed as brightness.

ii. Luminous flux: Luminous flux defines as how much light source emits (lm).

$1 \operatorname{lm}($ lumen $)=$ luminous intensity * steradian $\quad$ solid angle

iii. CCT (Color correlated temperature): It is the temperature of the blackbody radiator of which the its chromaticity point is closest to that of the chromaticity point of non-Planckian light source. CCT is normally represents the color of light such as bluish-white, reddish-white, or neutral.

\section{B. Color mixing technique}

This technique makes use of different LEDs with varying intensity of individual LED resulting in generation of white light. In color mixing technique, two LEDs are mixed together by varying the intensity in order to obtain the white light such as yellow mixed with blue.The asme technique can be used for three LEDs were $\operatorname{red}(\mathrm{R})$, green $(\mathrm{G})$, and blue(B) are mixed, or by using the four LEDs i.e $\operatorname{red}(\mathrm{R})$, green $(\mathrm{G})$, blue(B), and yellow (Y) (RGBY). In the proposed system the color mixing in achieved by using two bi-color LEDs strip that is warm white and cool white LEDs. Color mixing provides highly efficient outcome as compared to wavelength conversion. 
C. Calculation of CCT and luminous flux

In order to calculate the CCT using color sensor with given data from four channels: red, green, and blue, the response determine a particular source's CCT. The key aspect of acquiring this is to transfer the RGB responses to the chromaticity diagram is finding the point located on Planckian locus closest to source's chromaticity point.

The Chromaticity coordinates $(\mathrm{x}, \mathrm{y})$ are related to the tristimulus values named as (XYZ). However, to acquire a CCT,color sensor is used for light source to map the color sensor values (RGB) with a CIE tristimulus values. The first step includes in, mapping the sensor value (RGB) to the CIE2 tristimulus values (XYZ). The coordinates of chromaticity given as $(\mathrm{x}, \mathrm{y})$ can be obtained and calculated,therefore the CCT is expressed by McCamy's rule. Fig 2 gives an overall operational process to obtain CCT [16].

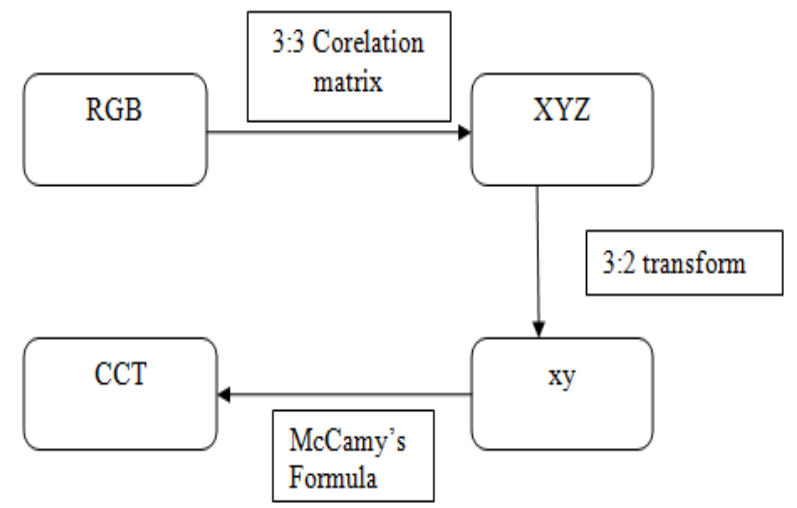

Fig. 2 Flow of CCT Calculation

The tristimulus values (XYZ) from CIE color space obtained in RGB values for color sensor and $(3 \times 3)$ Correlation Matrix as shown below in (1).

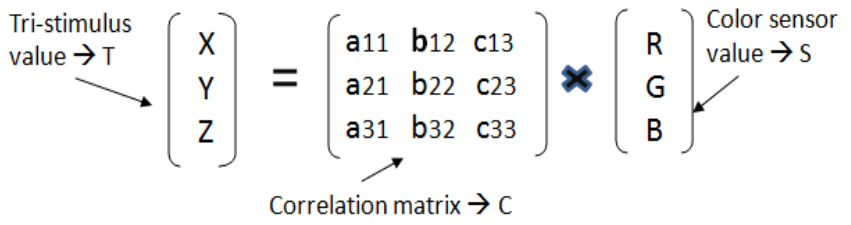

Once the tristimulus factors are regulated, the coordinates of coordinates can be readily calculated as follows.

$$
\begin{aligned}
& \mathrm{x}=\mathrm{X} /(\mathrm{X}+\mathrm{Y}+\mathrm{Z}) \\
& \mathrm{y}=\mathrm{Y} /(\mathrm{X}+\mathrm{Y}+\mathrm{Z})
\end{aligned}
$$

By Mc-Camy's rule, the CCT value can be expressed as in terms of the chromaticity coordinates [16].

$$
\begin{gathered}
\mathrm{CCT}=449 \mathrm{n}^{3}+3525 \mathrm{n}^{2}+6823.3 \mathrm{n}+5520.33 \\
\mathrm{n}=(\mathrm{x}-0.3320) /(0.1858-\mathrm{y})
\end{gathered}
$$

Actual (absolute) values of CCT obtained from the color sensor can be checked against the target values of CCTby calibrated integrating sphere. While calculating the first measurement, either warm or cool white LEDs need to be in on state. Now, only the warm-white LED strings are in on state. Here, (XwYwZw) tristimulus factor from the integrating sphere and the $(\mathrm{RwGwBw})$ signals from the colorimeter are obtained. For cool-white, the metric will be same as warm white.Now, for related values of $(\mathrm{XcYcZc})$ and $(\mathrm{RcGcBc})$ are obtained. For the third measurement, the tristimulus Matrix $\mathrm{T}$ is given in correlation matrix named as $\mathrm{C}$ and a color sensor value matrix as $\mathrm{S}$, therefore can be written, $\mathrm{T}=\mathrm{CS}$. Here, multiplying $\mathrm{C}$ on both sides by $\mathrm{S}^{-1}$, so the $\mathrm{C}$ is represented as $\mathrm{C}=\mathrm{TS}^{-1}$ in (7).

$$
\left(\begin{array}{lll}
a 11 & b 12 & c 13 \\
a 21 & b 22 & c 23 \\
a 31 & b 32 & c 33
\end{array}\right)=\left(\begin{array}{lll}
X w & X c & X n \\
Y_{w} & Y_{c} & Y n \\
Z w & Z c & Z n
\end{array}\right) \quad \boldsymbol{x}\left(\begin{array}{ccc}
R w & R c & R n \\
G w & G c & G n \\
B w & B c & B n
\end{array}\right)
$$

After obtained the values of correlation matrix, the target CCT values is verified with the actual (absolute) value using the integrating sphere. Here, the set of RGB values from color sensor is checked.

For calculation of luminous flux, the tristimulusvalues XYZ gives Y expressed as illuminance (luminance) in [18]. The luminous flux represented as $\varphi$.Hence, $\mathrm{Y}$ is multiplied by $\mathrm{k}$, where $\mathrm{k}$ is proportionality constant and given as (8), 


$$
\varphi=\mathrm{Y}(\mathrm{k})
$$

Through calibration the kvalueis obtained using the sensor. Based on the first measurement for warm white LEDs are set on, the value of $\mathrm{Y}$ can be calculated by using $(9)$ as follows.

$$
Y=a 21 R+a 22 G+a 23 B
$$

As the values of $\mathrm{Y}$ and $\varphi$ are already available, constant $\mathrm{k}$ expressed in (8). Here,value of $\mathrm{k}$ is 0.137 which is average having higher deviation of 0.003 .

\section{PROPOSED SYSTEM}

The PWM dimming is carried out the LEDs which are used in large mainly in industries in order to keep the mood of workers happy. Lighting of LEDs provides automatic or manual controlling of light levels. These helps for benefical dimming of systems. Here, individual LEDs of warm with cool white are used. The change in the CCT remains constant then the bi-color LED is considered as ideal. Here, CCT and luminous flux are independent of each other. The deviation in actual CCT and flux compared with target CCT and flux is occurred mainly due to the variations in LED aging and ambient temperature The deviation in CCT for greater than value $\pm 200 \mathrm{~K}$, the color drift (variation) is discoverable normally throughnormal eyes, that is common forbi-color LEDs. However, due to the various parameters such as highly nonlinear and strong connectionmediate light levels, CCT (color correlated temperature), driving current variations, luminous flux, and LEDs temperature,hence, difficult to obtain accurate color control and dimming with linear system.

\section{A. Problem statement}

i. The linearity must be obtained from Microcontroller 3.3V to provide $0-10 \mathrm{~V}$ scaling with LED driver.

ii. Designing the accurate dimming and color mixing of Bicolor LED's using feedback technique in microcontroller using a color sensor having the LED driver range of $0-10 \mathrm{~V}$.

iii. The precise mixing of warm white and cool white LED is difficult as the color sensor angle and LED angle for capturing light should be linear.

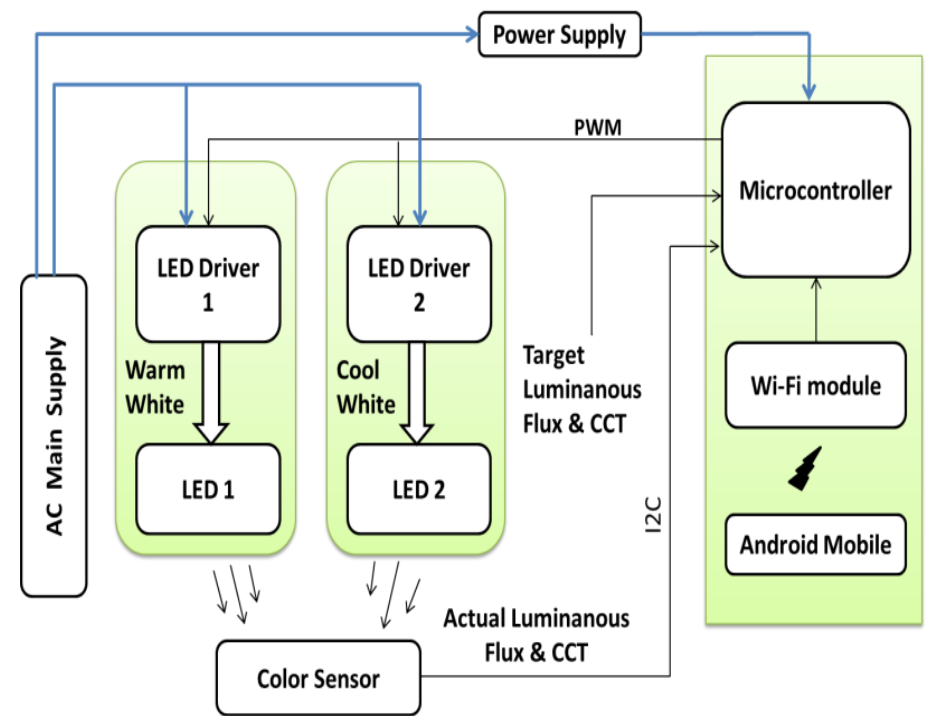

Fig. 3 System Block Diagram

Fig. 3 spectacles the fuctional diagram of corresponding system represented in [1].The color sensor monitors efficiently the light from two LED strings in which the output is bounded by closed loop entity such the each of them emits different values of CCT [17]. The sensor displays the digital outcome having red, blue and green (RGB) sensing values over microcontroller via the standard I2C serial bus using SCA and SCL bind.

LED driver is used to drive the LED with the scaling range of 0-10V. LED dimming can be obtained by either analog dimming or PWM dimming.The set of duty ratios (Dw, Dc) is used to regulate the tolerable currents independently through the cool and warm LED strings which generate the distinct PWM signals. A color sensor is embedded on the distant PCB in order to timely calculate CCT and luminous flux from both strings. Fed back in provided to the MCU such that the comparison is made between the actual (absolute) values of flux and CCT with target values of flux and 
CCT. By performing the devoted software methodology, a latest well-set of PWM ratios is determined by MCU for controlling the two warm white with cool white LED strings so the absolute (actual) values record the path nearly to the corresponding target CCT values and flux irrespective of changes in environmental conditions. Here, the closed-loop LED system shown below in fig. 5.

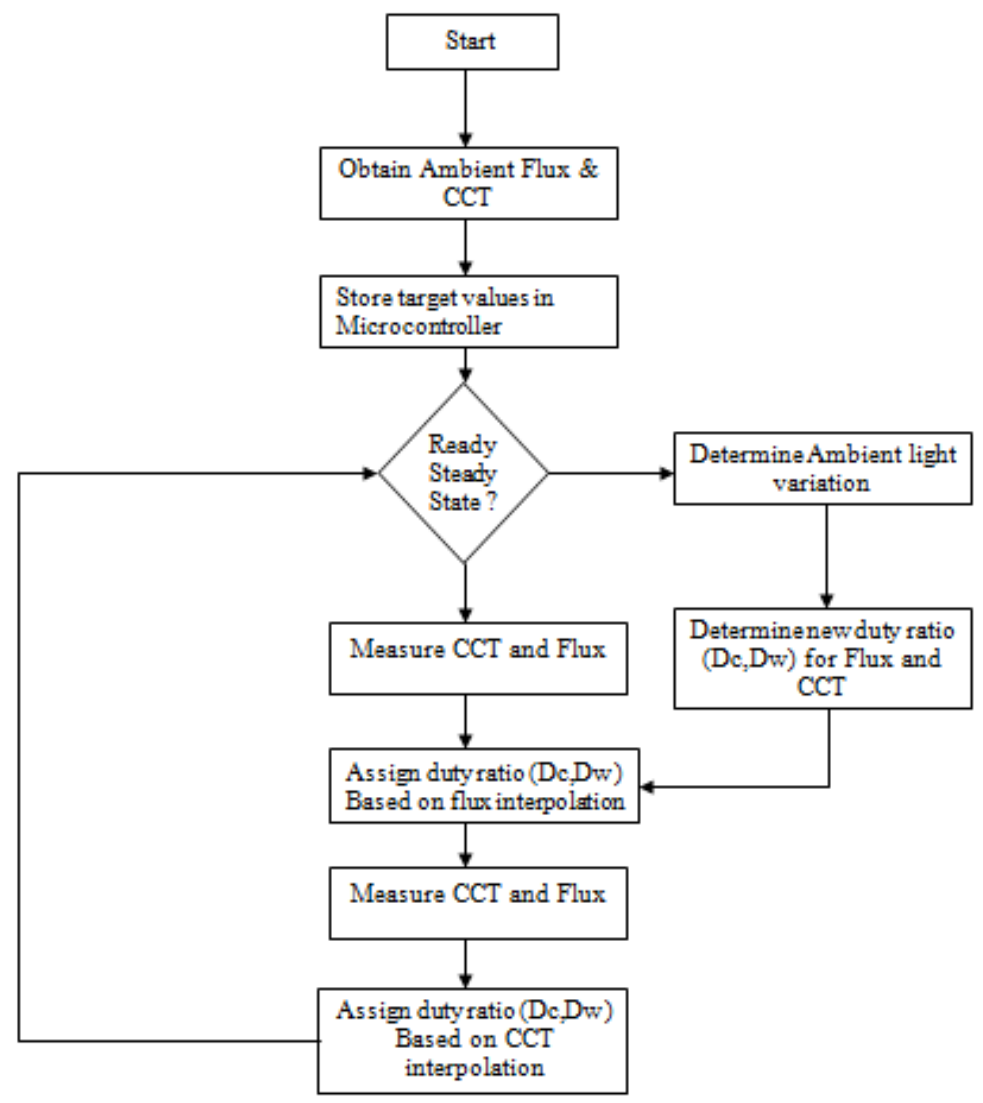

Fig.5 Flowchart for Closed loop Non-Linear system

By acquiring a nonlinear luminous along with CCT models [17], the duty ratios (Dw,Dc) can be obtained analytically. Here, it is impractical to obtain all possible values for complete set of luminous flux and CCT as the manipulation complexity and higher storage of memory will extent the growth of microcontroller.

\section{CONCLUSION}

The proposed system will provide the efficient dimming of warm white anlog with cool white using the closed loop non linear method. The color sensor will give the accurate values of RGB and CCT.The PWM dimming is will be in range of $0-10 \mathrm{v}$. In addition to it, the RGB LEDs can be used along with color sensor in order to provide the efficent PWMdimming through android mobile. The RGB LEDs will provide the original values which will be differnt from the RGB values in actual aspects.

\section{REFERENCES}

[1] Albert T. L. Lee, Member, IEEE, Huan-Ting Chen, Member, IEEE, "Precise Dimming and Color Control of Light-Emitting Diode Systems based on Color Mixing", IEEE Transactions on Power Electronics, Vol. 2, Issue 3, 2015.

[2] H. Chen, D. Y. Lin, S. C. Tan, and S. Y. Hui, "Chromatic, photometric and thermal modeling of LED systems with nonidentical LED devices", IEEE Trans. on Pow. Elect., vol. 29, no. 12. pp. 6636-6647, Dec. 2014.

[3] K. H. Loo, Y. M. Lai, S. C. Tan, and C. K. Tse, "On the color stability of phosphor-converted white LEDs under DC, PWM, and bilevel drive", IEEE Trans. on Power Elect., vol. 27, no. 2, pp. 974-984, Feb. 2012.

[4] F.-C. Wang, C.-W. Tang, B.-J. Huang, "Multivariable robust control for a red-green-blue LED lighting system", IEEE Trans. on Pow. Elect., vol. 25, no. 2, pp.417-428, Feb. 2010.

[5] X. Qu, S. C. Wong, and C. K. Tse, "Color control system for RGB LED light sources using junction temperature measurement", in Proc. IEEE Industrial Electronics Society (IECON), pp. 1363- 1368, Nov. 5-8, 2007.

[6] S. Muthu, F. J. Schuurmans, and M. D. Pashley, "Red, green, and blue LED based white light generation: issues and control"., Proc. Ind. Appl. Conf., pp. 327-333, Oct. 2002. 


\section{IJARCCE}

[7] M. Dyble, N. Narendran, A. Bierman, and T. Klein, "Impact of dimming white LEDs: chromaticity shifts due to different dimming methods", Proc. SPIE, vol. 5941, pp. 291-299, 2005.

[8] K. H. Loo, Y. M. Lai, S. C. Tan, and C. K. Tse, "Stationary and adaptive color-shift reduction methods based on the bilevel driving technique for phosphor-converted white LEDs"., IEEE Trans. on Pow. Elect., vol. 26, no. 7. pp. 1943-1953, Jul. 2011.

[9] M. M. Sisto, J. Gauvin, "Accurate chromatic control and color rendering optimization in LED lighting systems using junction temperature feedback", Proceedings of SPIE, vol. 9190, Sep. 2014.

[10] A. Lee, J. Sin, P. Chan, "Scalability of quasi-hysteretic FSM- based digitally controlled single-inductor dual-string buck LED driver to multiple strings", IEEE Trans. on Pow. Elect., pp. 501-513, vol. 29, no. 1, Jan. 2014.

[11] H. C. Kim, C. S. Yoon, H. Ju, D.-K. Jeong, J. Kim, "An AC-powered, flicker-free, multi-channel LED driver with current-balancing SIMO buck topology for large area lighting applications", in IEEE APEC, pp. 3337-3341, Mar. 2014.

[12] H. Chen, Y. Zhang, and D. Ma, "A SIMO parallel- string driver IC for dimmable LED backlighting with local bus voltage optimization and single time-shared regulation loop", IEEE Trans. on Pow. Elect., vol. 27, no. 1, pp. 452-462, Jan. 2012.

[13] W. Zhang, P. Sutardja, "Correlated color temperature control methods and devices", US Patent US20130020956 A1, Jan. 24, 2013.

[14] Peng Liu, et al., "Investigation of self-adaptive LED surgical lighting based on entropy contrast enhancing method", Optics Communication, vol. 319, pp. 133-140, May 2014.

[15] "Calculating color temperature and illuminance using the TAOS TCS3414CS digital color sensor", Designer's Notebook, pp. 1-7, Feb.2009[Online], Available: https://www.ams.com/ger/content/view/download/145158

[16] H.-T. Chen, et al., "Nonlinear Dimming and Correlated Color Temperature Control of Bi-Color White LED Systems".IEEE Trans. on Pow. Elect., Vol No. 4, Issue 5.

[17] M. Magno, T. Polonelli, L. Benini, and E. Popovici, "A low cost, highly scalable wireless sensor network solution to achieve smart led light control for green buildings", IEEE Sensors Journal, vol. 15, no. 5, pp.2963-2973, 2015.

[18] Gilbert held, "Introduction to light emitting diode technology and applications" www.auerbach-publications.com, New York,1943. 